\title{
Linear regression models for estimating true subsurface resistivity from apparent resistivity data
}

\author{
Sabiu Bala Muhammad ${ }^{1,2}$ and Rosli SaAd ${ }^{1, *}$ \\ ${ }^{1}$ School of Physics, Universiti Sains Malaysia, 11800 USM, Pulau Pinang, Malaysia. \\ ${ }^{2}$ Department of Physics, Usmanu Danfodiyo University, Sokoto, Nigeria. \\ *Corresponding author. e-mail: rosli28260@gmail.com
}

MS received 4 July 2017; revised 29 September 2017; accepted 22 November 2017; published online 25 June 2018

Simple linear regression (SLR) models for rapid estimation of true subsurface resistivity from apparent resistivity measurements are developed and assessed in this study. The objective is to minimize the processing time and computer memory required to carry out inversion with conventional algorithms. The arrays considered are Wenner, Wenner-Schlumberger and dipole-dipole. The parameters investigated are apparent resistivity $\left(\rho_{a}\right)$ and true resistivity $\left(\rho_{t}\right)$ as independent and dependent variables, respectively. For the fact that subsurface resistivity is nonlinear, the datasets were first transformed into logarithmic scale to satisfy the basic regression assumptions. Three models, one each for the three array types, are thus developed based on simple linear relationships between the dependent and independent variables. The generated SLR coefficients were used to estimate $\rho_{t}$ for different $\rho_{a}$ datasets for validation. Accuracy of the models was assessed using coefficient of determination $\left(R^{2}\right), F$-test, standard error $(S E)$ and weighted mean absolute percentage error $(w M A P E)$. The model calibration $R^{2}$ and $F$-value are obtained as 0.75 and 2286, 0.63 and 1097, and 0.47 and 446 for the Wenner, Wenner-Schlumberger and dipoledipole array models, respectively. The $S E$ for calibration and validation are obtained as 0.12 and 0.13 , 0.16 and 0.25 , and 0.21 and 0.24 for the Wenner, Wenner-Schlumberger and dipole-dipole array models, respectively. Similarly, the $w M A P E$ for calibration and validation are estimated as 3.27 and $3.49 \%, 3.88$ and $5.72 \%$, and 5.35 and $6.07 \%$ for the three array models, respectively. When compared with standard constraint least-squares (SCLS) inversion and Incomplete Gauss-Newton (IGN) algorithms, the SLR models were found to reduce about $80-96.5 \%$ of the processing time and memory space required to carry out the inversion with the SCLS algorithm. It is concluded that the SLR models can rapidly estimate $\rho_{t}$ for the various arrays accurately.

Keywords. Simple linear regression; true resistivity; apparent resistivity; processing time; memory space.

\section{Introduction}

Resistivity survey plays significant role in many large-scale sites characterization (Dahlin et al. 2007; Bery and Saad 2012), groundwater exploration (Saad et al. 2012a) and mineral prospecting
(Saad et al. 2012b; Song et al. 2017). Unfortunately, the measured apparent resistivity data from such surveys cannot be used directly to describe the subsurface (De Donno and Cardarelli 2017). Inversion is therefore required to develop resistivity models that can portray the true subsurface 
condition based on the apparent resistivity data. The necessary requirements for the inversion of any geophysical data include fast forward algorithm for calculating theoretical data for initial model parameters, and a technique for calculating derivatives of the data with respect to the model parameters (Narayan et al. 1999).

Resistivity inversion has received enormous attention from various researchers over the years. Investigations are still going on to further mitigate the problems associated with its speed and efficacy. Several researches have been conducted to develop and control resistivity inversion mechanisms. The least-squares optimization based techniques are the most commonly and widely explored compared to other approaches (Olayinka and Yaramanci 2000; Loke and Dahlin 2002; Loke and Lane 2004). They have been applied to solve many practical inversion problems of simple and complex structures with recorded remarkable success (Pain et al. 2002). They are, however, not without problems. Some of them include nonuniqueness of solutions, great consumption of time and memory space for the iterative computations to converge at minimum data misfit (Gyulai et al. 2010; Vachiratienchai and Siripunvaraporn 2013), inability to produce accurate models when the starting initial model is far from the actual solution, emergence of false anomalies at intermediate and final iterative computations of model resistivity (Liu et al. 2012) and difficulties in dealing with nonlinear relationships among the modelling (subsurface) parameters (Singh et al. 2010; Maiti et al. 2012; Srinivas et al. 2012; Stanley Raj et al. 2014).

Joint inversion technique attempts to address the problems of multiple solutions and false anomalies arising from resistivity inversion. It has performed well with additional data from seismic and ground penetrating radar (GPR) surveys (Zhou et al. 2014). Research algorithms that have this functionality are available nowadays, but possibilities to combine several geophysical methods in one inversion are limited. Survey time to acquire extra GPR or seismic data for large sites and picking the first arrival time to provide the needed datasets for the joint inversion are also other factor to consider (Bergmann et al. 2014). Finally, even though electrical resistivity and seismic velocity tomograms correlate with one another, very important anomalies as lateral changes in resistivity may not be detected by the seismic method, and can therefore be phased out as false anomalies.
Regression coefficients obtained from linear least squares method can provide alternative means for rapidly estimating the true subsurface resistivity whenever apparent resistivity site measurements are conducted. Nonlinearity in the nature underground resistivity distributions can be taken care of, by using logarithmic transform of datasets, rather than using the actual datasets directly in the regression process. The objective of this research is to develop and assess mathematical models for fast estimation of true subsurface resistivity from apparent resistivity measurements. This is expected to significantly reduce the time and memory capacity needed to process the apparent resistivity data using conventional algorithms. While acknowledging the applicability of linear regression method to solving many scientific problems, the originality of this research lies in its ability to first develop simple models for estimating true subsurface resistivity from apparent resistivity data by regression. This will provide the user with alternative means for rapidly estimating true resistivity without recourse to complex conventional inversion algorithms, thereby saving significant processing time and computer memory space.

\section{Study area}

This research was carried out at Universiti Sains Malaysia main campus, Pulau Pinang, Malaysia. Pulau Pinang (Penang Island) comprises an area of about $285 \mathrm{~km}^{2}$, connected to the mainland by two long bridges. The temperature typically, ranges from $29^{\circ}-32^{\circ} \mathrm{C}$, with the highest temperature occurring between April and June, every year. The average relative humidity ranges from 65-95\%, with lowest values recorded between the months of June and September. Average monthly rainfall may reach up to $240 \mathrm{~mm}$, and is not usually lower than $58.6 \mathrm{~mm}$ (Pradhan and Lee 2010). Measured resistivity in the area varied from 0 to $6000 \Omega \mathrm{m}$, depending on the nature of materials and degree of saturation (Abdulrahman et al. 2016).

Dominant geology is basically granite; classified according to the proportions of alkali feldspars to total feldspars (Ahmad et al. 2006). The northern part of the Island is abundantly characterized with alkali feldspars that contain orthoclase to intermediate microcline, they do not show any distinct cross hatched twining. In the southern part, however, they show well developed cross hatched twining and are characterized to be microcline 

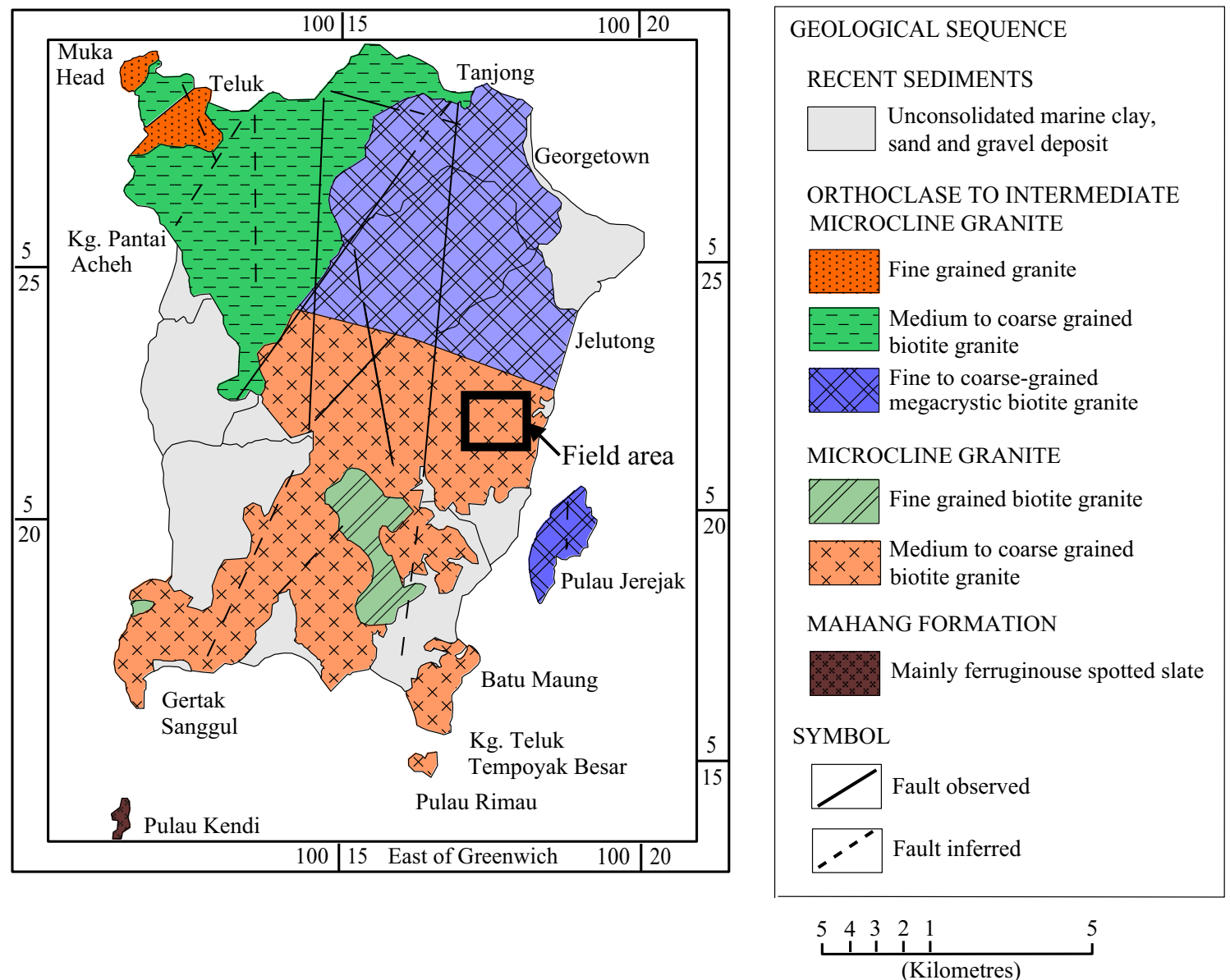

Figure 1. Geology map of Penang Island (modified from Pradhan and Lee 2010).

(Ong 1993). Penang Island is composed of a few soil types; clay materials of Kuala-Kedah, the steep land soil series, which is composed of weathered granitic rocks and is obtained at the central part of the island, fine to medium coarse sandy clay of Rengam series developed on igneous and high grade metamorphic rocks, and Selangor Kangkong association, which is primarily comprised of medium grained clay derived from acid sulphate soils. Alluvium and colluvium series, which constitute fine to medium grained loamy materials derived locally, also exist (Pradhan and Lee 2010). The geologic map of Penang Island showing the study area is given in figure 1 .

\section{Methodology}

\subsection{Resistivity data}

A typical electrical resistivity method measures underground potential contrast $(V)$ upon injecting specific amount of current $(I)$ into the ground (figure 2). Resistance $(R)$ is then automatically computed from Ohm's law (equation 1) and subsequently the apparent resistivity, when array geometrical factor is known.

The data utilized for the regression modelling was acquired from ground resistivity survey, using multi-electrode resistivity meter (ABEM Terrameter SAS4000), conducted along two profiles each for the Wenner-Schlumberger and dipole-dipole arrays; one for model calibration (line 1) and the other for model validation (line 2). For the Wenner array however, data along three profiles were acquired for model calibration (lines 1-3) and one profile for validation (line 4). This was necessary to ensure sufficient data for the modelling, as surveys with Wenner array produce less number of data points compared to the remaining two. Each line consisted of 41 stainless steel electrodes at $1 \mathrm{~m}$ minimum spacing connected to two smart cables, each of 21 take-outs, using jumper. The current was set between 1 and $100 \mathrm{~mA}$. A computer controlled system Terrameter SAS4000 was used to automatically select the four active electrodes 


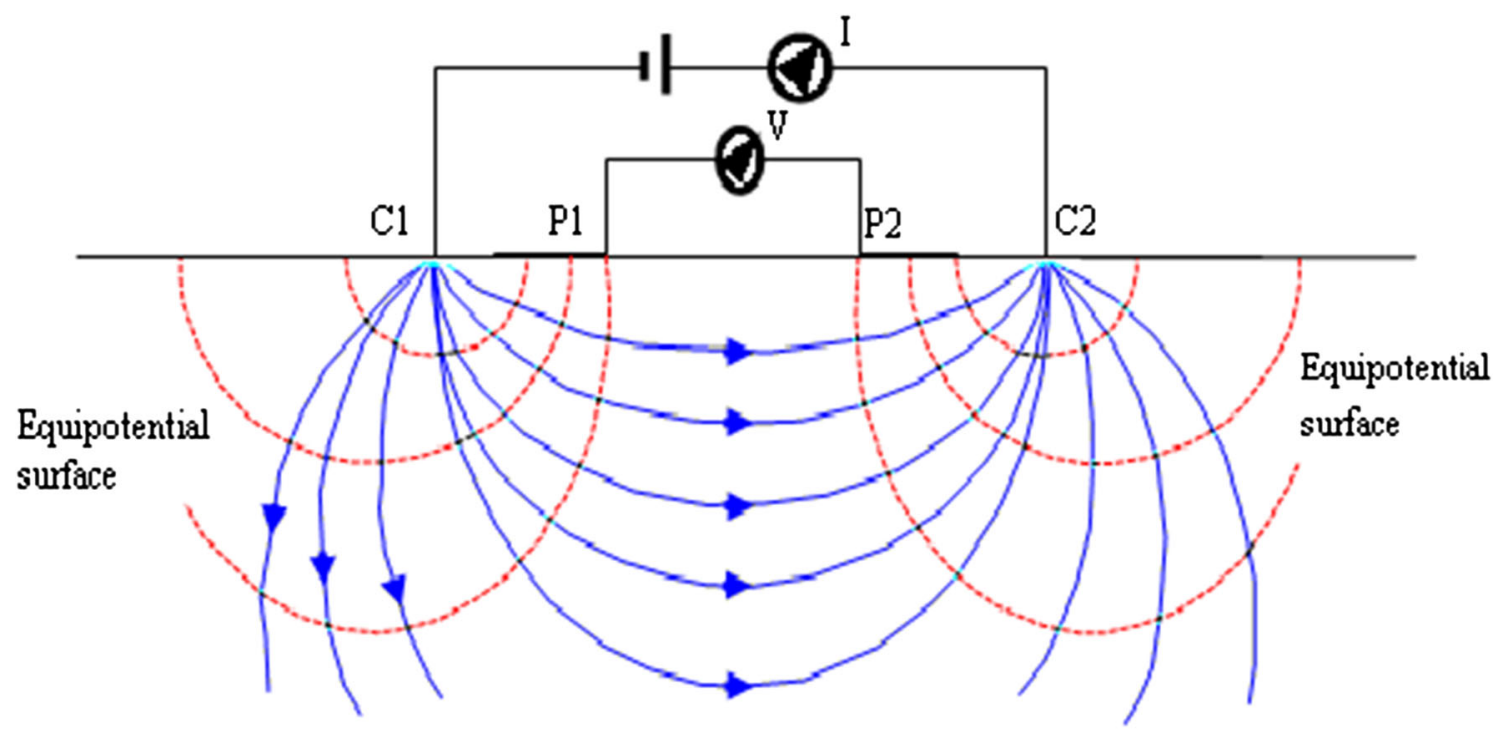

Cument lines

Figure 2. Four-point electrode configuration with current and potential distribution.

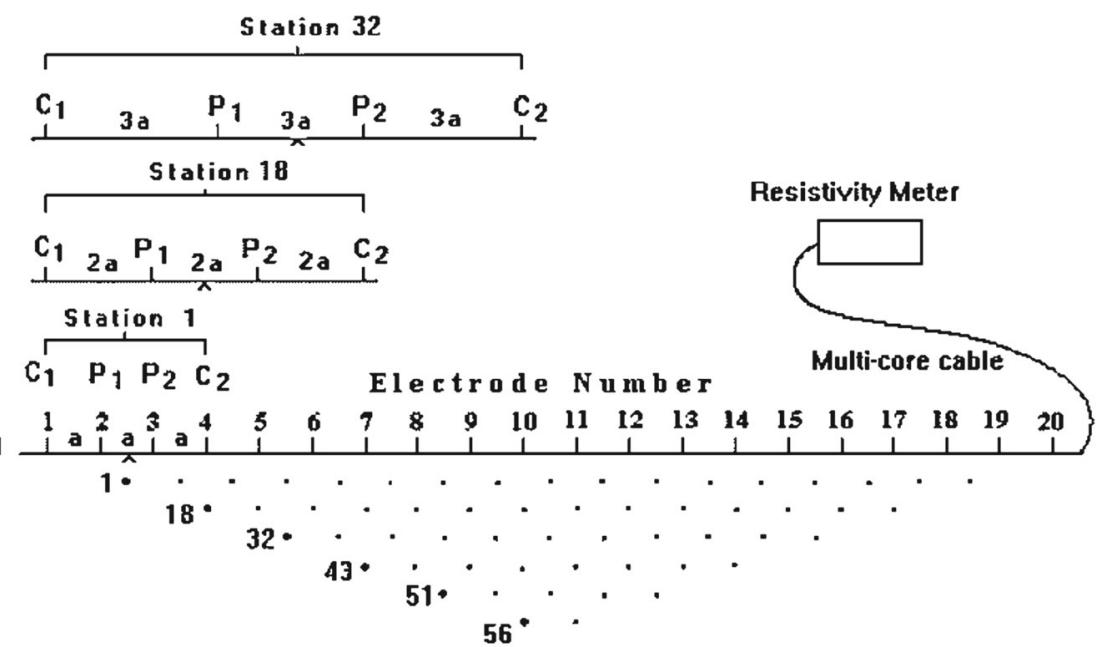

sequence of measurements to build up a pseudosection

Figure 3. Data points arrangement for a typical resistivity measurement.

for each voltage measurement and to consequently compute the apparent resistivity (equation 2 ) using the array geometrical factor $(k)$ for each array. Apparent resistivity data points were arranged systematically such that each datum point is specified with its horizontal and vertical coordinates (figure 3)

$$
\begin{aligned}
V & =I R, \\
\rho_{a} & =k \frac{\Delta V}{I} .
\end{aligned}
$$

All the measured apparent resistivity data were subjected to inversion using standard constraint least-squares (SCLS) inversion algorithm available in Res2Dinv software package, to obtain the true resistivity models and datasets $\left(\rho_{t}\right)$ at same coordinates as the apparent resistivity $\left(\rho_{a}\right)$. The datasets $\left(\rho_{a}\right.$ and $\left.\rho_{t}\right)$ were then arranged in columns in preparation for the linear regression modelling.

\subsection{Linear regression}

Generally, least squares based linear regression model with $n$ independent variables $(x)$ in the observations and a dependent variable $(y)$ can be written as: 


$$
y=\beta_{0}+\beta_{1} x_{1}+\beta_{2} x_{2}+\cdots+\beta_{n} x_{n}+\varepsilon
$$

where $\beta_{0}$ is the intercept, $\beta_{1}, \beta_{2}, \ldots, \beta_{n}$, are the regression coefficients and $\varepsilon$ is the error term. In this approach, the model converges when the squared sum of the differences between the estimated and observed values is minimum. The values of dependent variable can then be estimated for given values of independent variable(s), if the errors are assumed to be normally distributed with zero mean and constant variance. The distribution of the $y_{i}$ is thus symmetrical and bell-shaped, with a constant standard deviation at $x$ levels (Balarabe et al. 2016).

In this study, true resistivity $\left(\rho_{t}\right)$ is considered as the dependent variable, while apparent resistivity $\left(\rho_{a}\right)$ is the independent variable. Unfortunately, nonlinear nature of the subsurface resistivity restricted the use of datasets directly for the regression modelling. To overcome this, the datasets were transformed into logarithmic scale to satisfy the regression pre-modelling diagnostics such as; tests of linearity, normality of the dependent variable, normality and probability of standardized residuals, heteroscedasticity, axes balance and outliers. Logarithmic transforms of $\rho_{a}$ and $\rho_{t}$, denoted as $\rho_{A}$ and $\rho_{T}$, respectively, are therefore adopted as the new model parameters. Outliers were removed based on Mahalonobis, Cook's and Centred Leverage distances. A modified form of equation (3) can thus be written as equation (4) to fit the new model parameters. First dataset for the three arrays each was imported into SPSS to run the regression (model calibration). Regression coefficients obtained from model calibration were applied to on the second datasets to estimated $\rho_{T}$ values for validation. Thereafter, $\rho_{T}$ were transformed back to $\rho_{t}$ and plotted using Golden Surfer 11 software, at the same colour scale and blanking as the observed true resistivity models, for visual assessment.

The accuracy of each model was assessed using coefficient of determination $\left(R^{2}\right), F$-test, standard error $(S E)$ and weighted mean absolute percentage error $(w M A P E)$, given in equations (5-8), respectively for model calibration, and $S E$ and $w M A P E$ for validation

$$
\begin{aligned}
\rho_{T} & =\beta_{0}+\beta_{1} \rho_{A} \\
R^{2} & =\frac{\sum\left(\hat{\rho}_{T i}-\bar{\rho}\right)^{2}}{\sum\left(\rho_{T i}-\bar{\rho}\right)^{2}}
\end{aligned}
$$

$$
\begin{aligned}
F_{\text {val }} & =\frac{R^{2} /(K-1)}{\left(1-R^{2}\right)(N-K)} \\
S E & =\left[\frac{1}{N} \sum\left(\rho_{T i}-\hat{\rho}_{T i}\right)^{2}\right]^{1 / 2} \\
w M A P E & =\frac{1}{N} \sum\left|\frac{\left(\rho_{T i}-\hat{\rho}_{T i}\right)}{\rho_{T i}}\right| \times 100
\end{aligned}
$$

where $\rho_{T i}, \hat{\rho}_{T i}$ and $\bar{\rho}_{T i}$ are observed, estimated and average logarithms of true resistivity values respectively, $k$ is the number of independent variables and $N$ is the number of data points.

The performance of the new SLR models with respect to processing time and computer memory space consumed in data processing has been recorded and compared with other established algorithms to further validate the efficacy of the models. SCLS inversion was used as bench-mark, while the Incomplete Gauss-Newton (IGN) algorithm with data compression option, specifically designed to reduce processing time and computer memory space required for the inversion using SCLS technique especially when dealing with many data points, was then compared with the new SLR models.

\section{Results and discussion}

Figure 4 presents the analyses carried out to check the basic regression assumptions. Figure 4(a) shows that logarithmic transform of the true resistivity data is approximately symmetrical, suggesting a normally distributed dependent variable as against the asymmetrically distributed actual (untransformed) true resistivity data. The histograms depicted by figure $4 \mathrm{~b}$ show that the standardized residuals obtained from the transformed datasets are also approximately normally distributed, with symmetrical bell-shape and almost zero skewness and kurtosis, as compared to the asymmetrical right skewed standardized residuals of the actual dataset (Cramer 1998; Cramer and Howitt 2004; Doane and Seward 2011). The normal probability curve of expected cumulative probability against observed cumulative probability indicates a steady increase in expected cumulative probability for every increase in the observed cumulative probability, until a nearly straight-line curve is achieved for the transformed dataset (figure 4c). The untransformed data, on the other hand, did not show such linearity. 
Untransformed (actual) Resistivity dataset

(a)

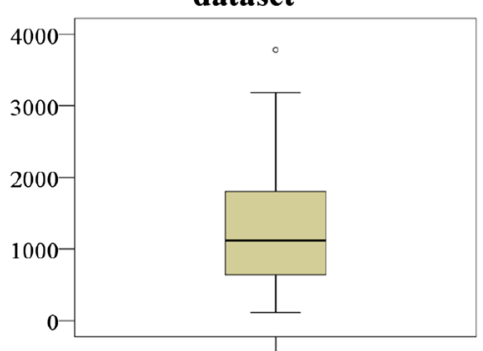

True Resistivity

(b)

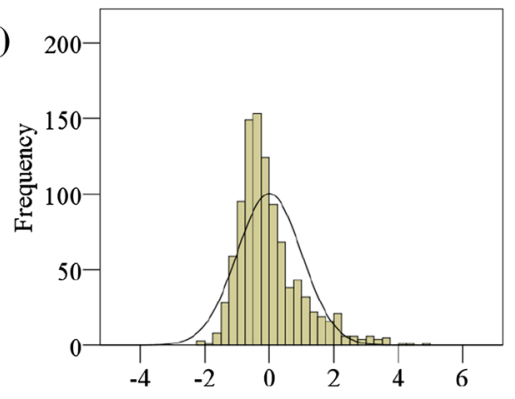

(c) Regression Standardized Residual

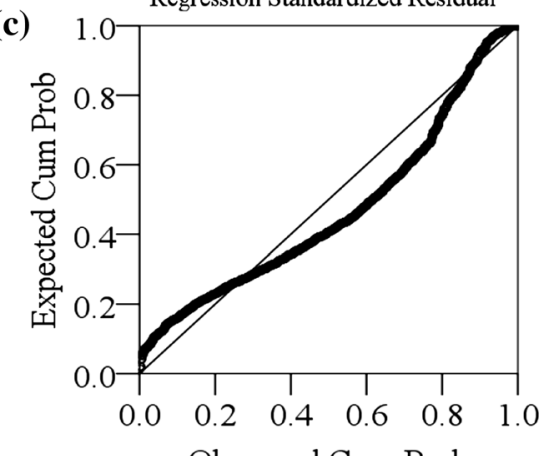

(d)

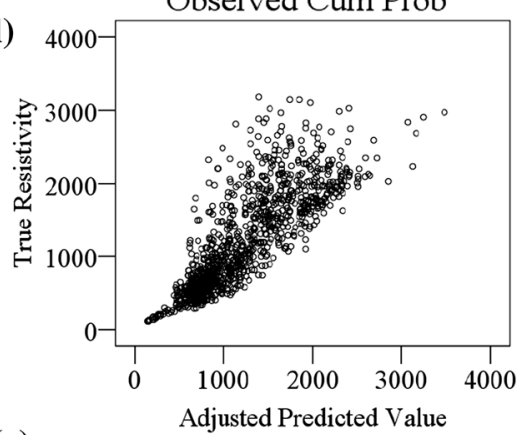

(e)

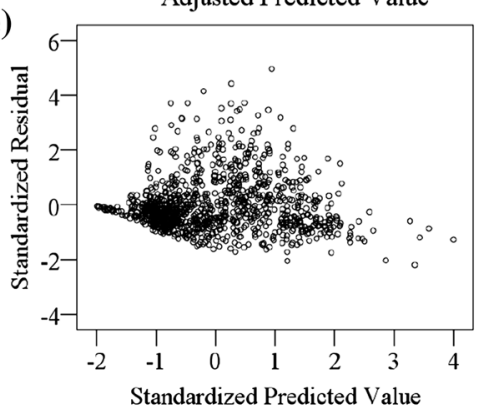

\section{Logarithm (transformed) Resistivity} dataset
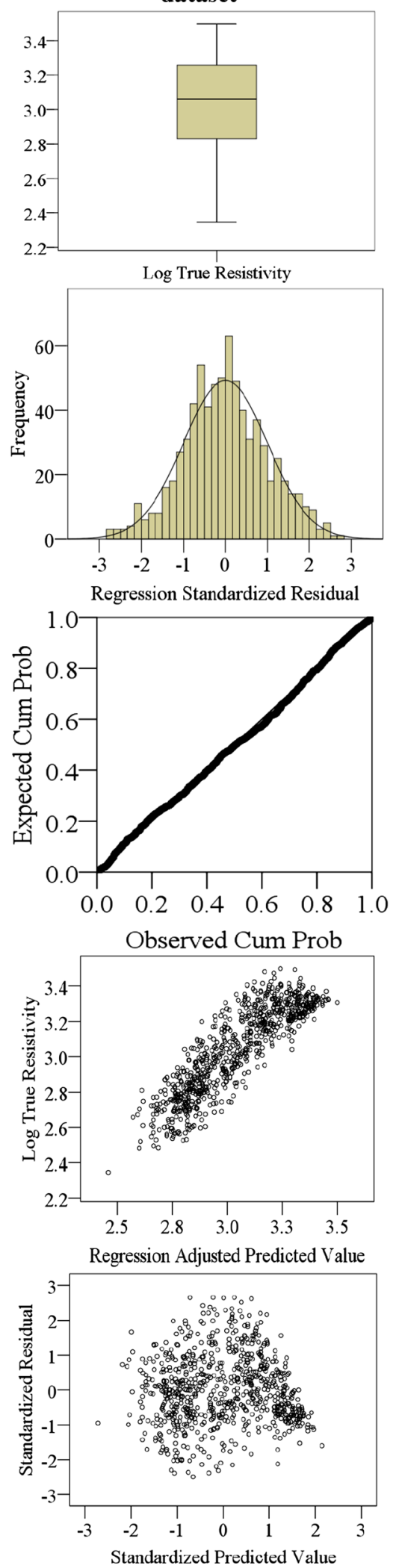

Figure 4. Basic regression assumptions checks (a) box plot, (b) histogram, (c) normal probability, (d) variables scatter plot, and (e) standardized residual scatter plot. 
Table 1. Models summary table.

\begin{tabular}{|c|c|c|c|c|c|c|c|c|}
\hline \multirow[b]{2}{*}{ Models } & \multicolumn{2}{|c|}{ Coefficients } & \multirow[b]{2}{*}{$R^{2}$} & \multirow[b]{2}{*}{$F$-value } & \multicolumn{2}{|c|}{$S E$} & \multicolumn{2}{|c|}{$w M A P E(\%)$} \\
\hline & $\beta_{0}$ & $\beta_{1}$ & & & $\mathrm{Cal}$ & $\overline{\mathrm{Val}}$ & Cal & Val \\
\hline Wenner & 1.22 & -0.60 & 0.75 & 2286 & 0.12 & 0.13 & 3.27 & 3.49 \\
\hline Wenner-Schlum & 1.14 & -0.36 & 0.63 & 1097 & 0.16 & 0.25 & 3.88 & 5.72 \\
\hline Dipole-dipole & 1.01 & 0.07 & 0.47 & 446 & 0.21 & 0.24 & 5.35 & 6.07 \\
\hline
\end{tabular}

Note. Cal $=$ Calibration, Val $=$ Validation, Wenner-Schlum $=$ Wenner-Schlumberger.

Figure 4(d) is the scatter plot of the dependent $v s$. independent variables of both actual and transformed datasets for linearity check. Visual assessment of both plots indicates that the transformed data points are approximately linearly distributed as compared to the nonlinear distribution of actual resistivity datasets. Similarly, the scatter plots of the standardized residuals suggest that all the residual data points of the transformed datasets are uniformly distributed between -3 and +3 on both axes with almost a zero mean, and without any clear pattern (figure 4e). On the other hand, residuals of the actual dataset are between -2 and +4 on horizontal axis, and between -4 and +6 on the vertical axis, with non-uniformity everywhere and a noticeable distribution pattern. All the pre-modelling assumption examined in this study have favoured the use of logarithmic transforms of datasets for the regression over the actual datasets.

Table 1 presents summary of the three regression models developed in this study. The generated coefficients $\beta_{0}$ and $\beta_{1}$, when substituted in equation 4 , will give the complete model required for estimating the true resistivity for each array type. The $R^{2}$ showcases how much variability in the dependent variable is explained by the independent variable. The $R^{2}$ is found to be $0.75,0.63$ and 0.47 for Wenner, Wenner-Schlumberger and dipole-dipole models, respectively. This signifies that $75 \%, 63 \%$ and $47 \%$ of variance in the true subsurface resistivity is attributed to the apparent resistivity derived from the three models, respectively. The remaining variance (\%), lumped as the model residuals, may be explained by the array sensitivity pattern and geological complexities. The highest value of $R^{2}$ obtained with Wenner array further indicates that the array is less affected by sensitivity pattern or other geological constraints (thereby depending mostly on the apparent resistivity measurement) to estimate the true resistivity. All the $R^{2}$ values obtained fall within strong to very strong range, according to the absolute strength classification of $R^{2}$ established by Evans (1996) and reported by Beldjazia and Alatou (2016) as: $<0.04$ (very weak), 0.04-0.15 (weak), 0.16-0.35 (moderate), $0.36-0.63$ (strong) and $0.64-1.00$ (very strong).

The $F$-test also compares the variance accounted for by each regression model (table 1). The null hypothesis of the test is defined in such a way that the regression models' coefficients are zero. The alternative hypothesis is that at least one of the coefficients is not zero, to allow for the response to be estimated. For the three models developed in this study, the $F$-values were found to be $F(1,750)=2286, p<0.001 ; F(1,637)=1097$, $p<0.001$; and $F(1,512)=446, p<0.001$ for the Wenner, Wenner-Schlumberger and dipole-dipole array models, respectively. The two numbers in parenthesis (degrees of freedom) indicate the number of independent variables and total number of data points, respectively. The $p$-values of less than $0.001(<0.1 \%)$ associated with each $F$-value further demonstrates the statistical significance of all the three models; as $p$-value of $0.05(5 \%)$ or less is required to reject the null hypothesis.

To further assess the models' accuracy to estimating the dependent variables, $S E$ for calibration and validation are respectively computed as 0.12 and $0.13,0.16$ and 0.25 , and 0.21 and 0.24 for Wenner, Wenner-Schlumberger and dipole-dipole array models, respectively. The calibration and validation $W M A P E$ are also estimated as 3.27 and $3.49 \%, 3.88$ and $5.72 \%$, and 5.35 and $6.07 \%$ for the three models, respectively (table 1 ). It is important to note that both $S E$ and $w M A P E$ for validation are slightly higher than the corresponding calibration errors. This is because outliers were not removed from the validation datasets as was the case for the calibration datasets. This was deliberately allowed for, since apparent resistivity field data are less frequently filtered for outliers before they are processed to obtain the true subsurface resistivity. Of the three models developed and assessed, the Wenner based model was found to be the most accurate, having the highest $R^{2}$ and $F$-value, with least $S E$ and $w M A P E$. 
Table 2. Comparison of the regression models' processing time, memory space consumed and error occurred with established algorithms.

\begin{tabular}{|c|c|c|c|c|c|c|c|c|}
\hline \multirow[b]{2}{*}{ Models } & \multicolumn{3}{|c|}{ Processing time (s) } & \multicolumn{3}{|c|}{ Memory (MB) } & \multicolumn{2}{|c|}{$w M A P E(\%)$} \\
\hline & SCLS & IGN & SLR & SCLS & IGN & SLR & IGN & SLR \\
\hline Wenner & 16.2 & 15.4 & 1.8 & 11.4 & 12.5 & 0.4 & 2.7 & 3.5 \\
\hline Wenner-Schlum & 24.2 & 25.8 & 2.2 & 12.3 & 12.8 & 0.6 & 4.1 & 5.7 \\
\hline Dipole-dipole & 27.0 & 27.5 & 2.3 & 12.5 & 13.2 & 0.7 & 4.7 & 6.1 \\
\hline
\end{tabular}

Note. SCLS = standard constraint least-squares inversion, IGN = incomplete Gauss-Newton with data compression option, SLR = simple linear regression.

(a)

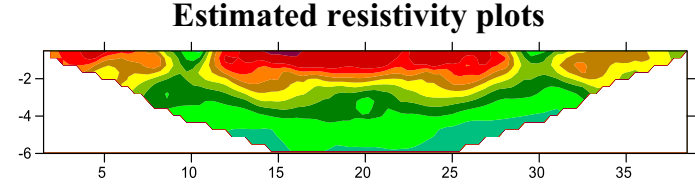

(b)

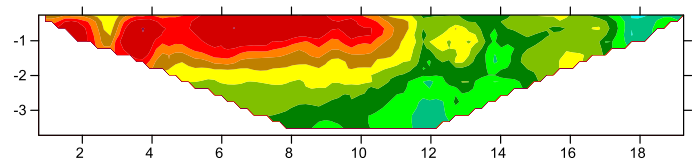

(c)

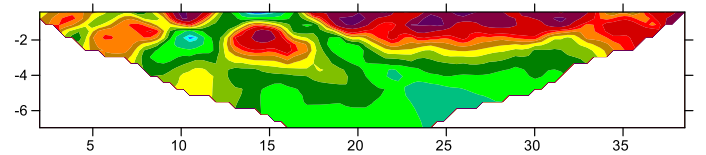

Observed resistivity plots
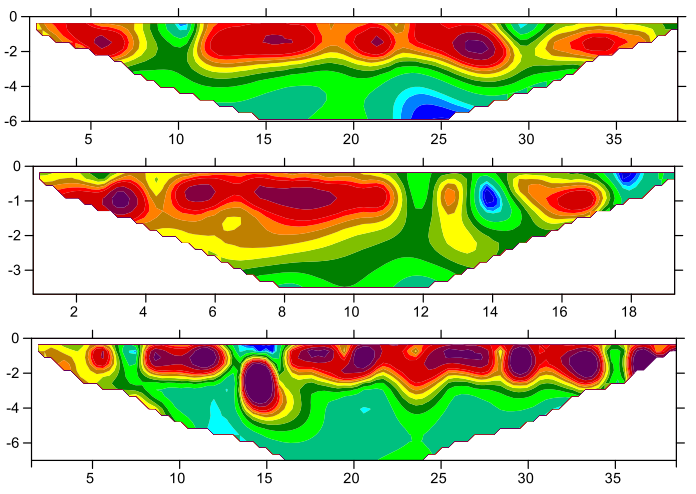
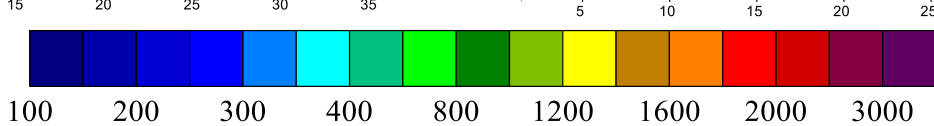

$1200 \quad 1600 \quad 2000 \quad 3000$

Figure 5. Comparison between estimated and observed resistivity contour plots (a) Wenner, (b) Wenner-Schlumberger, and (c) Dipole-dipole array models for visual assessment.

Table 2 provides summary of the regression models' processing time, memory space consumed and errors occurred in comparison with other established algorithms, namely SCLS and IGN. It took about $16.2,154.4$ and $1.8 \mathrm{~s}$, respectively for SCLS, IGN and SLR to process the validation data acquired using the Wenner array. The memory spaces consumed by the SCLS, IGN and SLR in processing the same data are $11.4,12.5$ and $0.4 \mathrm{MB}$, respectively. This shows that the SLR (Wenner) model is able can cut down up to $80 \%$ of the processing time and $96.5 \%$ of the computer memory space required by the SCLS algorithm to carry out the inversion. In comparison, the IGN algorithm brought about 5\% reduction in processing time and $10 \%$ increase in memory space consumed by the SCLS inversion algorithm. Comparable results were also obtained with the Wenner-Schlumberger array model. The SLR (Wenner-Schlumberger) model could save respectively 90 and $95 \%$ of the processing time and memory space consumed by the SCLS inversion algorithm, as compared to the IGN algorithm, which brought about respectively 6.6 and $4 \%$ increase in time and memory space.
Similarly, the dipole-dipole based SLR model has cut down respectively 91.5 and $94.4 \%$ of the processing time and memory space consumed by the SCLS algorithm. This is in contrast with the IGN algorithm with 1.9 and $5.6 \%$ increase in time and memory space, respectively. However, the SLR models recorded slightly higher wMAPEs compared to the IGN algorithm. The differences in WMAPEs are $0.8,2.6$ and $2.4 \%$ for the Wenner, Wenner-Schlumberger and dipole-dipole arrays, respectively. This may be a compromise between slightly more accurate true resistivity estimates and enormous reduction in processing time and memory space needed especially when dealing too many data points.

Two-dimensional contour plots of the estimated true resistivity derived from the validation datasets were constructed and compared to the corresponding observed true resistivity plots for visual assessment of the models (figure 5). For the Wenner (figure 5a) and dipole-dipole (figure 5c) arrays, the resistivity contour plots depict high resistivity zone (above $1200 \Omega \mathrm{m}$ ) above $3 \mathrm{~m}$ depth and low resistivity zone (below $1200 \Omega \mathrm{m}$ ) below the depth of about 
$3 \mathrm{~m}$. High resistivity region at the top left part of the tomogram produced using the observed data with Wenner-Schlumberger array is also noticeable in the estimated data plot (figure 5b). Both the estimated and observed data plots for the three arrays did not differ much from one another in terms of subsurface resistivity distribution, indicating that the models can be relied upon for satisfactory estimations. The Wenner based model has also been proven the best by the visual assessment.

\section{Conclusion}

Three simple linear regression models for rapid estimation of true subsurface resistivity from apparent resistivity data have been developed and validated in this study. Numerical accuracy assessment carried out suggests that all the $R^{2}$ values obtained fall within strong to very strong range, with Wenner model has the highest $R^{2}(0.75)$ and $F$-value (2286) with lowest $S E(0.12)$ and $w M A P E$ $(3.27 \%)$. Visual assessment using contour plots has also placed the Wenner model at the top of the list, having resistivity distribution closest to its corresponding observed plot. When compared with Standard constraint least-squares (SCLS) inversion and Incomplete Gauss-Newton (IGN) algorithms, the SLR models were found to curtail $80-96.5 \%$ of the processing time and memory space required to carry out the inversion with SCLS algorithm. It is therefore concluded that all the three models can rapidly estimate true resistivity whenever the corresponding arrays are used to acquire apparent resistivity data, with the Wenner model being the most accurate.

\section{Acknowledgements}

The authors would like to thank the technical staff and postgraduate students, Geophysics Section, School of Physics, Universiti Sains Malaysia, Pulau Pinang, Malaysia for their help during the data acquisition.

\section{References}

Abdulrahman A, Nawawi M, Saad R, Abu-Rizaiza A S, Yusoff M S, Khalil A E and Ishola K S 2016 Characterization of active landfill sites using $2 \mathrm{D}$ resistivity/IP imaging: Case studies in Penang, Malaysia; Environ. Earth Sci. 75(347) 1-17.
Ahmad F, Yahaya A S and Farooqi M A 2006 Characterization and geotechnical properties of Penang residual soils with emphasis on landslides; Am. J. Environ. Sci. 2(4) 121-128.

Balarabe M, Abdullah K, Nawawi M and Khalil A E 2016 Monthly temporal-spatial variability and estimation of absorbing aerosol index using ground-based meteorological data in Nigeria; Atmos. Clim. Sci. 6 425-444.

Beldjazia A and Alatou D 2016 Precipitation variability on the massif Forest of Mahouna (Northeastern-Algeria) from 1986 to 2010; Int. J. Manag. Sci. Bus. Res. 5(3) $21-28$.

Bergmann P, Ivandic M, Norden B, Rücker C, Kiessling D, Lüth S, Schmidt-Hattenberge C and Juhlin C 2014 Case History Combination of seismic reflection and constrained resistivity inversion with an application to $4 \mathrm{D}$ imaging of the $\mathrm{CO}_{2}$ storage site, Ketzin, Germany; Geophys. 79(2) B37-B50.

Bery A A and Saad R 2012 Clayey sand soil's behaviour analysis and imaging subsurface structure via engineering characterizations and integrated geophysicals tomography modeling methods; Int. J. Geosci. 3 93-104.

Cramer D 1998 Fundamental Statistics for Social Research: Step-by-step Calculations and Computer Techniques using SPSS for Windows; London: New York, Routledge.

Cramer D and Howitt D L 2004 The Sage Dictionary of Statistics: A Practical Resource for Students in the Social Sciences; Sage, London and Thousand Oaks, CA.

Dahlin T, Wisén R and Zhang D 2007 3D effects on 2D resistivity imaging-modelling and field surveying results; In: 13th European Meeting of Environmental and Engineering Geophysics, Istanbul, Turkey.

De Donno G and Cardarelli E 2017 Tomographic inversion of time-domain resistivity and chargeability data for the investigation of landfills using a priori information; Waste Manag. 59 302-315.

Doane D P and Seward L E 2011 Measuring skewness: A forgotten statistic; J. Stat. Educ. 19(2) 1-18.

Evans J D 1996 Straightforward statistics for the behavioural sciences; Brooks/Cole Publishing Company.

Gyulai Á, Ormos T and Dobroka M 2010 A quick 2-D geoelectric inversion method using series expansion; J. Appl. Geophys. 72(4) 232-241.

Liu B, Nie L, Li S, Xu L, Liu Z, Song J, Li L and Lin C 2012 3D electrical resistivity inversion tomography with spatial structural constraint. Yanshilixue Yu Gongcheng Xuebao/Chinese; J. Rock Mech. Geotech. Eng. 31(11) $2258-2268$.

Loke M H and Dahlin T 2002 A comparison of the GaussNewton and quasi-Newton methods in resistivity imaging inversion; J. Appl. Geophys. 49(3) 149-162.

Loke M H and Lane J W 2004 Inversion of data from electrical resistivity imaging surveys in water-covered areas; Explor. Geophys. 35(4) 266-271.

Maiti S, Erram V C, Gupta G and Tiwari R K 2012 ANN based inversion of DC resistivity data for groundwater exploration in hard rock terrain of western Maharashtra (India); J. Hydrol. 464-465 294-308.

Narayan S, Dusseault M B, Nobes and D C 1999 Inversion techniques applied to resistivity inverse problems; Inverse Prob. 10(3) 669-686. 
Olayinka A I and Yaramanci U 2000 Use of block inversion in the 2-D interpretation of apparent resistivity data and its comparison with smooth inversion; J. Appl. Geophys. 45(2) 63-81.

Ong W S 1993 The geology and engineering geology of Pulau Pinang; Jabatan Penyiasatan Kajibumi, Malaysia.

Pain C C, Herwanger J V, Worthington M H and De Oliveira C R E 2002 Effective multidimensional resistivity inversion using finite-element techniques; Geophys. J. Int. 151(3) 710-728.

Pradhan B and Lee S 2010 Delineation of landslide hazard areas on Penang Island, Malaysia, by using frequency ratio, logistic regression, and artificial neural network models; Environ. Earth Sci. 60(5) 1037-1054.

Saad R, Mohamad A S and Adli I 2012a 2-D resistivity and induced polarization (IP) methods for iron ore exploration; Electron. J. Geotech. Eng. 17 2973-2979.

Saad R, Nawawi M N M and Mohamad E T 2012b Groundwater detection in alluvium using 2-D electrical resistivity tomography (ERT); Electron. J. Geotech. Eng. 17(D) 369-376.

Singh U K, Tiwari R K and Singh S B 2010 Inversion of 2-D DC resistivity data using rapid optimization and minimal complexity neural network; Nonlin. Process. Geophys. 17(1) 65-76.

Song W, Wang Y and Shao Z 2017 Categorical modeling on electrical anomaly of room-and-pillar coal mine fires and application for field electrical resistivity tomography; $J$. Appl. Geophys. 136 474-483.

Srinivas Y, Stanley Raj A, Oliver D H, Muthuraj D and Chandrasekar N 2012 A robust behavior of Feed Forward Back propagation algorithm of artificial neural networks in the application of vertical electrical sounding data inversion; Geosci. Front. 3(5) 729-736.

Stanley Raj A, Srinivas Y, Oliver D H and Muthuraj D 2014 A novel and generalized approach in the inversion of geoelectrical resistivity data using Artificial Neural Networks (ANN); J. Earth Syst. Sci. 123(2) 395-411.

Vachiratienchai C and Siripunvaraporn W 2013 An efficient inversion for two-dimensional direct current resistivity surveys based on the hybrid finite differencefinite element method; Phys. Earth Planet. Inter. 215 $1-11$.

Zhou J, Revil A, Karaoulis M, Hale D, Doetsch J and Cuttler S 2014 Image-guided inversion of electrical resistivity data; Geophys. J. Int. 197(1) 292-309. 